\title{
proposición de pliego de condiciones y precios de contratación para hormigón sumimistrado en obra
}

JOSE M. LOPEZ SAIZ, ingeniero de caminos

\section{simapsis}

El presente artículo tiene como finalidad el exponer un posible Pliego de Condiciones para la contratación de hormigón premezclado, y promover entre los técnicos interesados en este tema una discusión de la cual pueda salir un Pliego de Condiciones adecuado a las necesidades actuales, ya que el volumen de contratación de este tipo de hormigón es muy grande, y en algunas ciudades, casi exclusivo, sin que se cuente con un Pliego que regule adecuadamente este tipo de suministro.

La primera parte del artículo es, simplemente, el citado Pliego de Condiciones; en la segunda parte se comentan las razones que han motivado algunos de los artículos, sobre todo en lo referido a modalidades de pago, así como se hace un comentario acerca del control de hormigón en obra, en desacuerdo con la Instrucción recientemente publicada por el M.o.P., intentando que en este punto exista también un intercambio de ideas entre los técnicos dedicados a esta rama de la tecnología. Por lo demás, se puede observar a lo largo del articulado que la presente proposición de Pliego de Condiciones sigue la actual "Instrucción» del M.O.P. (1968).

\section{Alcance}

El presente Pliego regulará el suministro, medición y pago de los hormigones preparados suministrados por en la obra

El hormigón suministrado habrá de cumplir ciertas condiciones de consistencia, resistencia y tamaño máximo del árido.

Estas condiciones se indicarán en el pedido expresando: el tipo hormigón a suministrar, mediante una clave compuesta de una letra que indicará la consistencia; un guión, una serie de números que serán la resistencia característica exigida a 28 días en probeta cilíndrica; una raya inclinada y otra serie de números, que indicarán el tamaño máximo del árido en milímetros.

Los diferentes descensos del cono pedidos se indicarán por las letras:

$$
\begin{array}{ll}
D=\text { Consistencia dura. } & \text { Descenso del cono hasta } 4 \mathrm{~cm} . \\
P=\text { Consistencia plástica. } & \text { Descenso del cono de } 4 \text { a } 8 \mathrm{~cm} . \\
B=\text { Consistencia blanda. } & \text { Descenso del cono de } 8 \text { a } 12 \mathrm{~cm} . \\
F=\text { Consistencia fluida. } & \text { Descenso del cono de } 12 \text { a } 18 \mathrm{~cm} .
\end{array}
$$

Por tanto, un hormigón con descenso del cono de 4 a $8 \mathrm{~cm}$, con una resistencia característica exigida a 28 días de $300 \mathrm{kp} / \mathrm{cm}^{2}$, y un tamaño máximo del árido de $35 \mathrm{~mm}$, se denominará:

$$
\text { P-300/35 . }
$$


A estos hormigones se les determinará un precio, el cual quedará especificado para cada uno de los tipos de hormigón de posible suministro en el contrato del que este Pliego es un anejo.

\section{Materialles}

Los materiales a emplear cumplirán las condiciones especificadas en la «Instrucción para el proyecto y la ejecución de las obras de hormigón armado y en masa» del M.O.P. (1968) para áridos y agua y en el «Pliego de Condiciones para la recepción de aglomerantes hidráulicos», en lo que respecta al cemento.

Podrá exigir ensayos de los materiales para demostrar que cumplen las condiciones especificadas; estos ensayos serán por cuenta de la empresa suministradora y podrán efectuarse a máximo de un ensayo cada $500 \mathrm{~m}^{3}$ de hormigón suministrado.

Inicialmente se efectuarán los ensayos necesarios para definir la calidad de los materiales y se presentarán por la empresa suministradora las dosificaciones a emplear y el tipo de cemento, al ingeniero jefe de la obra.

El suministro de hormigón se efectuará por la modalidad de resistencia; por ello, la empresa suministradora tiene libertad para variar la dosificación cuantas veces crea necesario para la mejor calidad del hormigón, respetando los tamaños máximos del árido y las consistencias especificadas, con la única condición de notificar al ingeniero jefe de obra de las variaciones efectuadas.

\section{Maquíncarion a emplear}

El hormigón puede fabricarse completamente en central y transportarse hasta el lugar de entrega en camiones agitadores o dosificarse en plantas especiales, mezclándose luego en camiones durante su transporte.

La capacidad de los camiones mezcladores será la indicada por el fabricante, sin poder superar las capacidades siguientes expresadas como porcentaje del volumen máximo de cabida del tambor:

- Camiones mezcladores para mezclado durante el transporte, $58 \%$.

- Camiones agitadores para transporte de hormigón mezclado en planta, $80 \%$.

En el caso de mezclado sobre camión durante el transporte, puede llegarse a un volumen del $68 \%$ del máximo del tambor si se garantiza que se obtiene un mezclado adecuado sin segregaciones.

\section{Welocidad de mezclade}

En los camiones mezcladores de tambor rotatorio esta velocidad estará comprendida entre 4 y $21,30 / D$ r.p.m., estando dado $D$ en metros. La velocidad de agitación no ha de ser menor de 2 ni mayor de 6 r.p.m.

\section{Tiempo de mezcliado}

El tiempo de mezclado comenzará a contar cuando todos los elementos, a excepción de la última porción de agua, estén en la mezcladora. 
4.1. Cuando el hormigón se mezcla en planta, el tiempo de mezclado será de 1 minuto para los primeros $3 / 4 \mathrm{de}^{3}$ de capacidad de la mezcladora. Cuando el volumen de la hormigonera exceda esta cantidad, se aumentará el tiempo de mezclado en 20 segundos por cada $\mathrm{m}^{3}$ o fracción.

El hormigón mezclado se transporta a obra y ha de descargarse antes de $1 \frac{1 / 2}{2}$ horas o de 300 revoluciones del tambor. Este tiempo de $1 \frac{1}{1 / 2}$ horas se disminuirá en tiempo cálido y podrá aumentarse cuando las condiciones sean favorables, a criterio del jefe de obra.

4.2. Si el hormigón se mezcla totalmente sobre el camión, el número de revoluciones a velocidad de mezclado dependerá del volumen de amasado.

Si ésta es igual a la capacidad teórica de mezclado, se efectuarán de -70 a 100 revoluciones.

Si ésta es menor que la citada capacidad, por lo menos en $0,5 \mathrm{~m}^{3}$, el número de revoluciones estará comprendido entre 50 y 100 .

Todas las revoluciones que excedan de 100 habrán de efectuarse a velocidad de agitación.

La operación de mezclado empezará dentro de los 40 minutos después de que se han entremezclado el cemento y los áridos. Una vez en el lugar de descarga y antes de proceder a la misma, se habrán de efectuar de 6 a 12 revoluciones a velocidad de mezclado.

Para lograr una consistencia uniforme, el número de revoluciones a velocidad de mezclado y de agitación se regulará para que resulten razonablemente uniformes de camión a camión.

4.3. En el caso de emplearse camiones mezcladores y siempre que el tiempo de transporte desde la planta a la obra no exceda de 40 minutos, se autoriza el transporte del hormigón sin giro del tambor durante la marcha, efectuándose el mezclado total una vez en obra. Para las velocidades de mezclado y número de vueltas necesario (máximo y mínimo), se tendrán en cuenta las prescripciones anteriores.

4.4. La descarga habrá de efectuarse siempre a plena abertura del dispositivo de descarga, y a ser posible a plenas revoluciones del tambor; si el ritmo de descarga así establecido es demasiado rápido por las condiciones de puesta en obra, la velocidad de descarga se regulará, únicamente, variando la velocidad del tambor o de las paletas de agitación.

Queda prohibida la adición de agua al tambor fuera de la planta de mezclado o de dosificación, salvo que en cada caso sea autorizado directamente por el jefe de obra. Cuando tal circunstancia especial se presentara, la descarga no se efectuará hasta que el tambor haya realizado por lo menos 20 revoluciones a velocidad de mezclado.

4.5. La temperatura del hormigón en el momento de la recepción en la obra no podrá ser superior a los $31^{\circ}$, ni inferior a los $4^{\circ} \mathrm{C}$, comprometiéndose la empresa suministradora a tomar las medidas necesarias para que estos límites de temperatura sean respetados.

\section{Aditivos}

Se autoriza el empleo de aditivos para mejorar las condiciones del hormigón, siempre que el tipo de los mismos y su dosificación sea conocida y aprobada por el ingeniero jefe de obra.

\section{Fomtrol de thommigón}

Se efectuará por su consistencia, tamaño máximo del árido y por su resistencia a compresión a 28 días en probeta cilíndrica de $15 \mathrm{~cm}$ de diámetro y $30 \mathrm{~cm}$ de altura. 


\section{Fabricución de Ins probetas}

Los moldes deberán ser metálicos y suficientemente rígidos para que no se deformen con el uso.

Para la ejecución de las probetas se separará la fracción del árido de tamaño superior a $50 \mathrm{~mm}$, mediante cribado por el cedazo 50 UNE 7050.

Después de homogeneizada la muestra se vierte en los moldes en capas de 10 a $12 \mathrm{~cm}$, y una vez bien distribuidos, el hormigón se compactará por apisonado o vibrado, si el hormigón es vibrado en obra, o por simple picado, en caso contrario, hasta que refluya el mortero y se produzca en la superficie una humectación brillante. Ejecutada de esta forma la primera probeta, las restantes se compactarán en cada capa con un número de golpes igual al empleado en la primera.

Una vez compactada la probeta se espolvorea la cara libre con cemento, enrasando nuevamente hasta conseguir una cara limpia y lisa.

Las probetas se desmoldearán a las 24 horas, manteniéndose durante ese tiempo cubiertas con sacos húmedos en lecho de arena o en cámaras apropiadas para evitar su desecación.

Se anotará la temperatura ambiente del lugar de ejecución de las probetas.

La probeta se marcará indicando la fecha de ejecución y los datos suficientes para su posterior identificación, en cuanto al tipo de hormigón y zona de obra en que se colocaron las amasadas a que corresponde la muestra.

Las probetas se conservarán hasta su rotura en ambiente húmedo o en agua a temperatura $20 \pm 2^{\circ}$ centígrados.

Si las probetas han de transportarse desde el lugar de ejecución al de rotura, habrán de hacerlo en recipientes adecuados rodeados de arena para evitar golpes, vibraciones, etc.

La rotura se efectuará en prensas debidamente contrastadas y a velocidad de carga de 3 a $7 \mathrm{kp} / \mathrm{cm}^{2} /$ segundo.

\section{B. Ejecución del contral}

El jefe de obra podrá adoptar el sistema que considere más apropiado teniendo en cuenta que se habrá de romper un mínimo de UNA probeta a 7 días y TRES a 28.

Como norma general se elegirá uno de los dos métodos de control que se proponen [a) y $b$ )], para los cuales se ejecutarán:

a) SEIS probetas de cada tipo de hormigón, rompiéndose TRES a 7 días y TRES a 28.

b) NUEVE probetas de cada tipo de hormigón, rompiéndose TRES a 7 días y SEIS a 28.

Se tomará como resistencia del hormigón controlado por una serie de probetas, a la media de las resistencias a 28 días de la serie; a este valor le llamaremos «resistencia de control».

Se denominará recorrido de una serie, a la diferencia entre los valores máximo y mínimo de las tensiones de rotura de las probetas de la serie. 
A partir de estos valores se formarán los gráficos de control siguientes:

Grafico 1. Gráfico de las resistencias de control de las series ensayadas para cada tipo de control.

Gráfico 2. Gráfico de las variaciones de la media de las resistencias de control en $N$ series consecutivas.

Grafico 3. Gráfico de la media de DIEZ recorridos consecutivos.

Estos mismos gráficos se llevarán, a efectos de control, con las resistencias a 7 días, sirviendo para tomar decisiones en lo que respecta a variaciones de dosificación, etc., para garantizar la obtención a 28 días de las resistencias contratadas.

El valor de $N$ depende de la definición escogida para la resistencia característica. Por ello, si el valor de $\alpha$ (definido más adelante) es $1, N$ toma el valor 6 ; si $\alpha$ vale $1,64, N$ vale 3 ; es decir:

$$
\begin{array}{ll}
\alpha=1 & \quad N=6 . \\
\alpha=1,64, & N=3 .
\end{array}
$$

\section{Dbtención de las probetas}

Las probetas de cada serie se obtendrán a la salida del camión-hormigonera una vez normalizado el proceso de descarga, con excepción de tratarse de hormigón bombeado, en cuyo caso las probetas se ejecutarán con hormigón obtenido a la salida de las tuberías.

Si la homogeneidad del hormigón dentro de un mismo camión-hormigonera no fuera suficiente a juicio del jefe de obra, podrá ordenar la obtención de la serie en tres grupos de DOS o TRES probetas, según el método de control elegido, tomando estos grupos uno al comienzo de la descarga, otra durante la misma y la tercera a su terminación.

\section{Resisstemcion contreatude}

La resistencia contratada se refiere a la resistencia característica del hormigón suministrado, que se obtiene de las resistencias de control mediante la fórmula siguiente:

siendo:

$$
R_{\text {c. obt }}=R_{m}-\alpha \cdot S \text {, }
$$

$\mathrm{R}_{\mathrm{c} . \mathrm{obt}}=$ Resistencia característica obtenida.

$R_{m}=$ Resistencia media de las resistencias de control de las series ejecutadas con ese tipo de hormigón.

$\alpha=$ Coeficiente de valor 1,64 .

$S=$ Desviación típica de las resistencias de control consideradas, la cual se obtiene de la fórmula:

$$
S=\frac{1}{n} \sqrt{n\left(R_{1}^{2}+R_{2}^{2}+R^{2}+\ldots R_{n}^{2}\right)-\left(R_{1}+R_{2}+R_{3}+\ldots R_{\mathrm{n}}\right)^{2}},
$$

siendo:

$n=$ número de series ejecutadas con el tipo de hormigón;

$R_{1}, R_{2}, R_{3} \ldots R_{n}=$ resistencias de control de las series $1,2,3 \ldots n$.

Con la autorización del ingeniero jefe de obra se podrá obtener la resistencia característica mediante métodos gráficos sobre papel probabilístico o por métodos estadísticos aproximados. 


\section{Medición y pago}

La medición se efectuará en la forma indicada en el contrato al que este documento queda anejo, y a esa medición se aplicarán los precios indicados en dicho contrato afectados de los coeficientes $C$ que más adelante se indican, caso de que la resistencia característica obtenida sea menor que la exigida.

\section{Eoeficientes a aplicar para la obtemción de los precios definitives [Bx]}

Se definirá como coeficiente de variación $(V)$ para todo lo que sigue al cociente:

Desviación típica del conjunto de resistencias de control del hormigón estudiado dividido por la resistencia media $\left(V=S / R_{m}\right)$.

Se distinguirán dos casos:

a) Coeficiente de variación menor o igual al $15 \%(V<0,15)$.

b) Coeficiente de variación mayor del $15 \%(V>0,15)$.

12.1. Caso $a) \quad(V \gtrless 0,15)$

La resistencia característica mínima será:

$$
R_{\mathrm{c}, \min }=0,8 R_{c},
$$

siendo:

$R_{c}=$ resistencia característica exigida; y considerándose el valor $R_{\mathrm{c} \text { m } \min }$ como resistencia de rechazo según definiremos más adelante.

Para resistencias características entre los valores $R_{c}$ y $R_{\mathrm{c} \text { m min }}$, los precios de contrato vendrán afectados por un coeficiente que se obtiene de la forma siguiente:

Se calcula el valor de $X$ mediante la siguiente fórmula:

$$
X=\frac{8\left(R_{c}-R_{\mathrm{obt}}\right)}{0,2 R_{c}},
$$

en donde:

$R_{\mathrm{obt}}=$ Resistencia característica obtenida en el conjunto de series del hormigón estudiado.

$R_{c}=$ Resistencia característica exigida a ese tipo de hormigón y $R_{\mathrm{c} \text {. min }}$ ya ha sido definida anteriormente.

A partir de este valor de $X$ se obtendrá el coeficiente a aplicar, cuyo valor será:

$$
C_{x}=1-0,021 X-0,013 X^{2} .
$$

\subsection{Caso $b) \quad(V>0,15)$}

En este caso, en las distintas fórmulas haremos intervenir el coeficiente de variación, por ello:

$$
R_{c . \min }=\frac{1-V}{A-B \cdot V} \cdot R_{c}=K_{\alpha} \cdot R_{c}
$$


siendo:

$$
\begin{gathered}
\alpha=1,64 \quad, \quad A=1,5 \quad \text { y } \quad B=3,75, \\
X=\frac{8\left(R_{c}-R_{\mathrm{obb}}\right)}{\left(R_{c}-R_{\mathrm{c} . \min }\right)}, \\
C_{x}=1,00-0,021 X-0,013 X^{2} .
\end{gathered}
$$

12.3. Por otra parte, en el caso $a)(V \gtrless 0,15)$, el coeficiente $C_{x}$ podrá ser aumentado hasta el valor de 1 a criterio del ingeniero jefe de obra si considerase que hay razones que justifiquen ese aumento; en el caso $b)(V>0,15)$, estos coeficientes serán de aplicación automática.

El valor de $C_{x}$ nunca podrá ser mayor que la unidad.

\section{Control dîario}

Como control para prever la posibilidad de resistencias características del conjunto, menores que la exigida, se estudiarán diariamente los gráficos de control en los cuales, si la resistencia característica del conjunto de resultados es igual o mayor que la exigida, ocurrirá:

1. Nunca se obtendrán más de dos resistencias de control consecutivas de valor menor que la resistencia característica exigida.

2. La media de las resistencias de control en TRES series consecutivas no será nunca menor que la resistencia característica exigida.

Para lograr que estas condiciones se cumplan, el jefe de obra advertirá a la empresa suministradora de las deficiencias que vayan apareciendo, siendo obligación de ésta tomar las medidas necesarias para su corrección. Caso de que estas medidas no fueran tomadas, tras dos advertencias consecutivas el ingeniero jefe de obra queda autorizado para penalizar con una multa de hasta el $10 \%$ de su precio a los hormigones cuyas resistencias de control no hayan alcanzado la resistencia exigida, considerándose que los ensayos efectuados en un día representan al hormigón suministrado durante ese día. Esta multa será independiente y acumulativa a la penalización correspondiente a la resistencia característica del conjunto, caso de ser aplicable.

Dado que no se pueden conocer las resistencias características de los diferentes tipos de hormigón hasta finalizado el suministro, se irán facturando las cantidades suministradas a los precios del contrato, de forma provisional.

Una vez terminado el suministro se procederá a la obtención de $C_{x}$, y aplicando este coeficiente al precio del hormigón y a los metros cúbicos suministrados se obtendrá la cantidad a pagar, y de ella el abono a efectuar por la empresa suministradora, la cual, para hacer frente a esta eventualidad, tendrá depositada una fianza, o será motivo de una retención equivalente al 10 ó al $15 \%$ de la cantidad a suministrar, con cargo a la cual se deducirán las penalizaciones a que hubiere lugar.

\section{Rechuzo de los hormigomes}

Caso de que la resistencia característica obtenida no alcanzara la $R_{\text {c. min }}$ antes definida, el hormigón se rechazará, siendo de cuenta de la empresa suministradora el costo de cuantas medidas fuere necesario tomar para garantizar la seguridad de las obras ejecutadas con ese hormigón. 
De igual forma pueden rechazarse partidas de hormigón suministradas durante uno o varios días cuando este hormigón no tenga resistencia suficiente, demoliendo o reforzando las zonas afectadas y siendo de cuenta de la casa suministradora los gastos de demolición y nueva ejecución, o los refuerzos, en su caso.

Serán motivo de rechazo opcional la obtención de resistencias de control de valor menor que $0,75 R_{c}$.

Será motivo de rechazo automático la obtención de resistencias de control de valor de $0,66 R_{c}$.

Caso de poder efectuarse el rechazo opcional, éste queda a criterio del ingeniero jefe de obra, quien, sin embargo, no deberá rechazar el hormigón si los resultados obtenidos en el conjunto hacen suponer que éste puede ser de suficiente calidad para el lugar en que ha sido colocado.

Para el tipo de rechazo opcional ha de tenerse en cuenta que un solo resultado no es suficiente para tomar una decisión.

\section{Competencius}

Para todo lo indicado en el presente Pliego serán decisorias en el siguiente orden de prelación y en cuanto no se opongan a las condiciones anteriormente expuestas:

La «Instrucción para el proyecto de obras de hormigón en masa y armado», del M.O.P. 1968.

La «Instrucción para el proyecto y ejecución de las obras de hormigón en masa y armado», del M.O.P. 1968.

Las «Recomendaciones prácticas unificadas para el cálculo y ejecución de las obras de hormigón armado», del C.E.B.

La Instrucción H.A. 61 del Instituto Eduardo Torroja.

Las Normas UNE.

Las restantes Normas del I.E.T.c.c.

Las Normas ACI números 214-64 y 318-67.

El criterio del ingeniero jefe de obra.

\section{Eomentamios}

Nos limitaremos a comentar los artículos 10, 12, 13 y 14, indicando las hipótesis que se han efectuado y los motivos de la obtención de las fórmulas indicadas. Debido a que la Instrucción del M.O.P. 1968 no entrará en vigor hasta el año 1971, se indicarán también las fórmulas de aplicación para el caso de definición de la resistencia característica del hormigón como lo hace la Instrucción H.A. 61; en este caso el valor de $\alpha$ (art. +10$)$ sería: $\alpha=1$.

Por la definición de resistencia característica y de coeficiente de seguridad podemos expresar las siguientes igualdades entre $R_{c}, R_{m}$ y resistencia de cálculo $R_{\text {calc }}$ :

$$
\begin{gathered}
R_{c}=R_{m}(1-\alpha \cdot V), \\
R_{\text {calc }}=\frac{R_{c}}{\gamma \cdot b}=\frac{R_{m}(1-\alpha \cdot V)}{\gamma \cdot b}=R_{m}[1-f(V, \alpha) V],
\end{gathered}
$$


teniendo en cuenta que $\gamma$ es función de $\alpha$ :

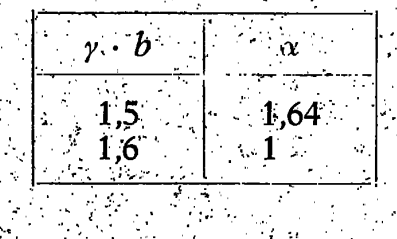

Y el valor de $f(V, \alpha)$ para distintos valores de $V$ y $\alpha$ es:

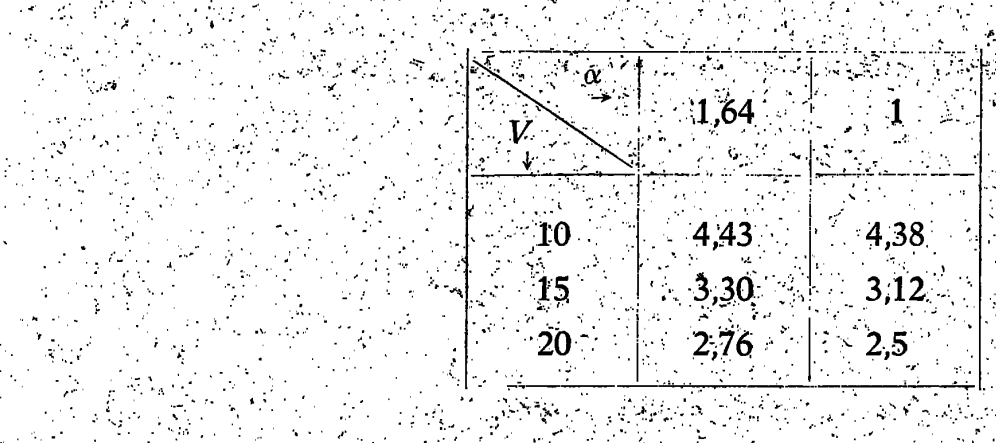

Y por tanto suponiendo una distribución normal para las resistencias de control y tomando un valor del coeficiente de yariáción del $15 \%$, náximo admisible en un hormigón debidamente controládo, calculamós las siguientes probabilidades de obtener un valor por debajo de la resistencia de cálculo (en el conjunto del hormigón de resistencia $R_{c}$ y coeficiente de variaciön $V=0,15$ ) función de $\alpha$ y que se expresa en là siguiente tabla:

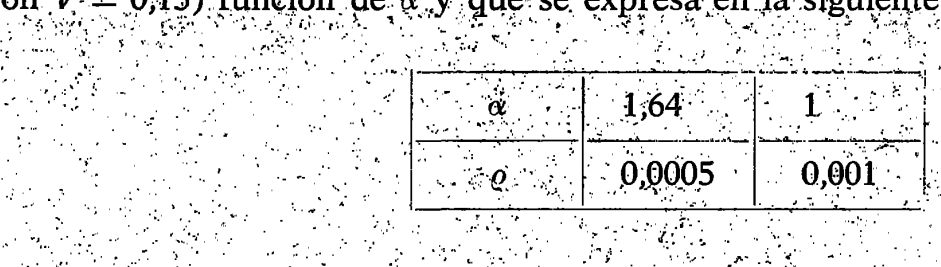

Como debemós establecer un criterio para obtener una resistencia característica mínima admisible para el hormigón, cóntratado con una résistenciá característica dada adinitiremos una variación de la resistencia del horningón en obra, dentro de unós ciértós limites, controlando là probabilidad de que las resistencias de cada amasada nó sean inferiôres a la resistencia de cálculö Para ello suponemos admisible una probabilidad dièz veces mayor que la obtenida si el hormigón tiene la resistencia carácterística exigida, és decir:

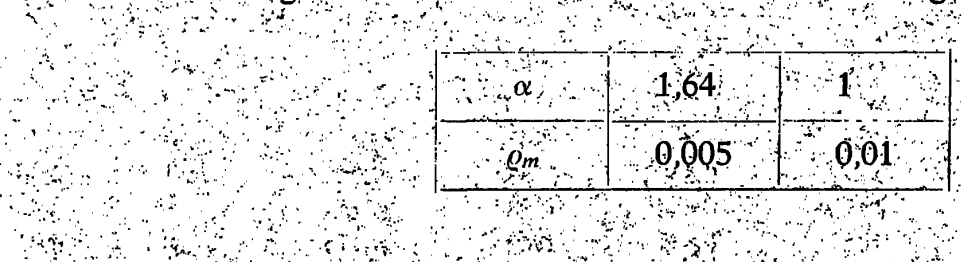

Resultan entônces, para valor de lâresistençấ ninima admisible, los vălores siguientes, función de la resistencia media obtenida en la serre de valores estudiados:

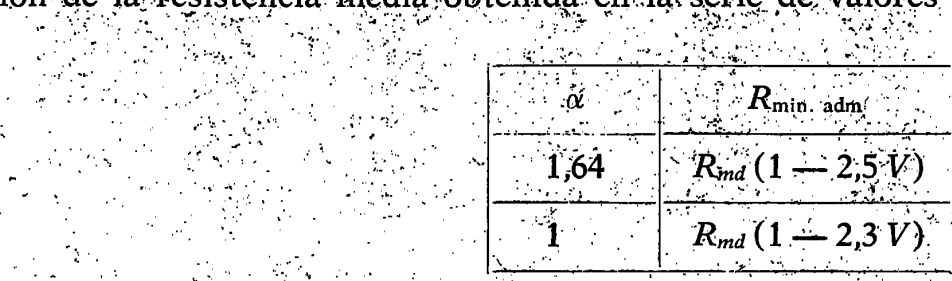


Y relacionando estos valores con la resistencia característica exigida tenemos:

$$
\begin{gathered}
R_{\mathrm{c} . \mathrm{obt}}=R_{\mathrm{m} . \mathrm{obt}}(1-\alpha \cdot V), \\
R_{\mathrm{m} . \mathrm{obt}}=\frac{R_{\mathrm{c} . \mathrm{obt}}}{1-\alpha \cdot V} ;
\end{gathered}
$$

de [1] y [3] se obtiene:

$$
R_{\min }=\frac{R_{\text {c. obt. } \min }(1-\beta \cdot V)}{1-\alpha \cdot V} ;
$$

ahora bien, como esta resistencia mínima ha de ser igual a la de cálculo:

$$
R_{\min }=R_{\text {calc }}=\frac{R_{c}}{b}
$$

igualando [5] y [4]:

$$
\frac{R_{c}}{\gamma \cdot b}=\frac{R_{\text {c. obt. } \min }(1-\beta \cdot V)}{1-\alpha \cdot V},
$$

y de ahí se obtiene la resistencia característica mínima admisible:

$$
R_{\text {c. } \min }=\frac{R_{c}(1-\alpha \cdot V)}{\gamma \cdot b(1-\beta \cdot V)}=\frac{R_{c}(1-\alpha \cdot V)}{A-B \cdot V}=K_{\alpha} \cdot R_{c},
$$

en la cual:

$A$ y $B$ son función a su vez de $\alpha$ (como lo es $\beta$ ) y tienen los siguientes valores:

\begin{tabular}{|c|c|c|c|c|c|c|c|c|}
\hline$V$ & $K_{1,64}$ & $K_{1}$ & $V$ & $K_{1,64}$ & $K_{1}$ & $V$ & $K_{1,64}$ & $K_{1}$ \\
\hline 0,15 & 0,804 & 0,810 & 0,18 & 0,854 & 0,875 & 0,21 & 0,920 & 0,955 \\
\hline 0,1525 & 0,808 & 0,816 & 0,1825 & 0,859 & 0,881 & 0,2125 & 0,927 & 0,963 \\
\hline 0,155 & 0,812 & 0,821 & 0,1856 & 0,864 & 0,887 & 0,215 & 0,933 & 0,971 \\
\hline 0,1576 & 0,816 & 0,826 & 0,1875 & 0,869 & 0,893 & 0,2175 & 0,940 & 0,979 \\
\hline 0,16 & 0,820 & 0,831 & 0,19 & 0,874 & 0,899 & 0,22 & 0,947 & 0,987 \\
\hline 0,1625 & 0,824 & 0,836 & 0,1925 & 0,879 & 0,906 & 0,2225 & 0,954 & 0,995 \\
\hline 0,165 & 0,828 & 0,841 & 0,1950 & 0,885 & 0,912 & 0,225 & 0,961 & 1,004 \\
\hline 0,1675 & 0,832 & 0,846 & 0,1975 & 0,890 & 0,919 & 0,2275 & 0,969 & 1,013 \\
\hline 0,17 & 0,836 & 0,852 & 0,20 & 0,896 & 0,926 & 0,23 & 0,977 . & 1,022 \\
\hline 0,1725 & 0,841 & 0,857 & 0,2025 & 0,902 & 0,933 & 0,2325 & 0,985 & - \\
\hline 0,175 & 0,845 & 0,863 & 0,205 & 0,908 & 0,940 & 0,235 & 0,993 & - \\
\hline 0,1775 & 0,850 & 0,869 & 0,2075 & 0,913 & 0,948 & 0,2375 & 1,002 & - \\
\hline- & - & - & - & - & - & - & - & - \\
\hline
\end{tabular}

\begin{tabular}{|l|c|c|}
\hline \multicolumn{1}{|c|}{$\alpha$} & $A$ & $B$ \\
\hline 1,64 & 1,5 & 3,75 \\
1 & 1,6 & 3,68 \\
\hline
\end{tabular}

A partir de estos valores se pueden tabular los de $K$, con lo que se obtiene la tabla I, a partir de $V=0,15(15 \%)$, ya que este valor del $15 \%$ se considera como el máximo obtenible con un buen control:

T A B L A I 
Como se puede observar, el valor de $K_{\alpha}$ para $V=0,15$ está muy próximo a 0,8 , y éste es el que se ha tomado por coincidir a su vez con el valor mínimo citado en la "Instrucción» del M.O.P. 1968; sin embargo, se puede ver que para valores de $V$ por encima de 0,151 lega a sobrepasar la unidad, es decir, que para coeficientes de variación altos puede llegar a ocurrir que ni la resistencia característica exigida sea suficiente para lograr que la probabilidad de no obtener en el hormigón resistencias menores que la de cálculo, alcance los valores mínimos indicados anteriormente. Por ello, en la propuesta del Pliego de Condiciones se diferencian los casos $V \gtrless 0,15$ y $V>0,15$.

Al mismo tiempo se han expresado fórmulas de pago, mediante las cuales se penaliza el hormigón caso de no haber obtenido la resistencia deseada.

A este respecto, y antes de pasar a hablar con más detalle de este coeficiente $C_{x}$ haremos una pequeña disgresión para expresar desacuerdo con la forma de control prevista en la «Instrucción para el proyecto...» del M.O.P. 1968. Esta Instrucción, en su artículo 234, regula la forma de efectuarse el control del hormigón puesto en obra, y dice: «se determinará la resistencia característica $\sigma_{b k}$ correspondiente a cada conjunto de al menos dos lotes consecutivos (es decir, sobre un mínimo de seis probetas), la cual debe resultar igual o superior a la exigida».

Desde el punto de vista estadístico no se pueden obtener valores confiables de la resistencia característica con sólo seis medidas (seis probetas), hacen falta al menos 25 observaciones (1). Pero es que aún hay otro error implicado en este sistema de control, y es que las tres probetas correspondientes a uno de los lotes (efectuados de una misma amasada) no definen la calidad del hormigón que se está produciendo, sino exclusivamente son tres medidas de un único valor que es la resistencia del hormigón en esa amasada y, por tanto, su variación es debida únicamente a las condiciones de ensayo (es igual que al medir una pieza para un muestreo, si esta medida se efectúa varias veces los valores obtenidos son distintos; esto no quiere decir que la pieza tenga medidas distintas, sino que el sistema de medida tiene una cierta función de distribución con su media y su desviación típica correspondiente y que indicará la bondad de la medición, pero no la medida ni la calidad de la pieza).

Por ambas razones creemos que el sistema de control propuesto no es estadístico ni adecuado al conjunto de la Instrucción. El sistema a seguir debe ser el de estudiar el conjunto total de resistencia del hormigón de cada tipo ejecutado bajo las mismas condiciones teóricas, es decir, siempre que se haya mantenido la dosificación, relación agua/cemento, etc., y, por tanto, las variaciones de resistencia no tengan una «causa asignable». Las resistencias de cada amasada no se tienen en cuenta más que para el cálculo de la resistencia característica y coeficiente de variación del conjunto, con excepción de que estos valores estén por fuera de la media más o menos tres veces la desviación típica, en cuyo caso hay que buscar una causa asignable para la obtención de tal resistencia.

Por otro lado, el control actual de, la «Instrucción» dice (art. 23.4, párrs. 3 y sigs.): «... Si no es así (si la resistencia no es mayor o igual que la exigida) se procederá a la realización de ensayos de información, etc. Si estos ensayos ofrecen resultados satisfactorios se aceptará la obra realizada:

- Cuando, por el contrario, la resistencia obtenida continúe siendo inferior a la exigida pueden considerarse dos casos.

- Si la resistencia deducida de las probetas testigo examinadas resulta superior al $80 \%$ de la resistencia característica exigida en el proyecto se realizarán pruebas de carga.»

Condiciones todas éstas que representan una exigencia de resistencia mínima para cada una de las amasadas, agravándose por la circunstancia de que esta resistencia mínima no es la media sino menor que la media.

Evidentemente, un estudio estadístico ha de efectuarse sobre el conjunto de resultados homogéneos. En todo lo expresado anteriormente se está de acuerdo con tomar como resistencia

(1) Cf. J. García Roselló: «Estudios y ejecución de hormigones para presas». Rev. O. P., agosto 1967, p. 913. 
mínima el valor de $0,8 R_{c}$; ahora bien, esto respecto al conjunto total de resultados. Si la resistencia obtenida para el total de un tipo de hormigón no llega a la resistencia característica exigida, habrá que sacar testigos o efectuar una prueba de carga, pero no en un lugar determinado, porque en esa zona o en ese elemento la resistencia de las probetas sea inferior a la característica exigida, sino en lugares elegidos totalmente al azar. La razón es sencilla:

1. La resistencia del hormigón de una amasada está representada por la media de la resistencia de las probetas ejecutadas de las mismas.

2. Si esta resistencia es mayor que la del cálculo, y teniendo en cuenta que además existe un coeficiente de seguridad a las cargas es evidente que la pieza ejecutada, si está debidamente calculada tiene que resistir necesariamente.

Por ello no parece adecuado ensayar en un lugar en el que se sabe de antemano cuál va a ser el resultado. Hay que hacer un ensayo al azar, buscando esas resistencias menores que la de cálculo, que tiene una cierta probabilidad en aparecer, mayor cuanto más se diferencia la resistencia característica obtenida de la exigida y mayor cuanto más grande es el coeficiente de variación.

Caso aparte merece la circunstancia de que alguna amasada dé como resistencia de control un valor menor que: $1^{\circ}$, la resistencia de cálculo, ó $2 .^{\circ}$, que la $R_{m}(1-3 V)$.

En el primer caso, el elemento ejecutado con ese hormigón debe ser demolido; en el segundo, hay que buscar una causa asignable a la obtenida de tal resultado, y uná vez encontrada habrá que ejecutar controles adecuados (obtención de testigos, prueba de carga) a todo el hormigón puesto en obra sobre el que han incluido estas circunstancias especiales (siempre que la causa no sea debida al ensayo, transporte, curado de las probetas... etc.; es decir, siempre que la causa sea imputable al hormigón ejecutado).

Por todas estas razones parece lo adecuado efectuar ensayos o pruebas de carga al azar en el conjunto de la obra, ensayos que han de cumplir a su vez ciertas condiciones para que den resultados confiables estadísticamente.

Esta es la razón por la cual al obtener resistencias características menores que las exigidas se conviene una penalización, cuyo importe se dedicará a las pruebas y ensayos necesarios para la confiabilidad en la obra ejecutada.

La penalización se ejecuta en forma de coeficiente reductor, función de una variable $X$ que lo es a su vez de la resistencia característica exigida, de la resistencia mínima admitida a ese hormigón (función del coeficiente de variación) y de la resistencia característica obtenida.

Esta variable $X$ toma la forma de la figura 1:

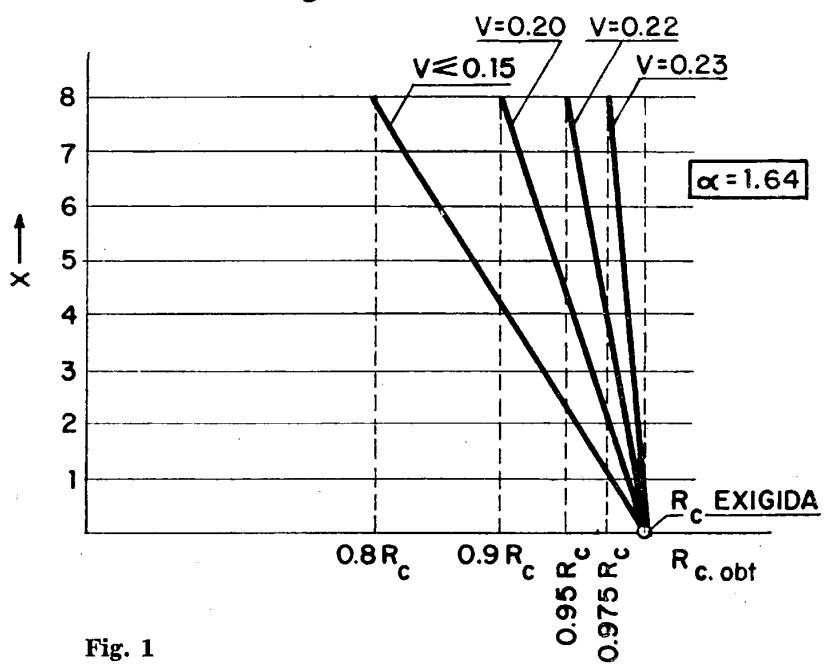


Y función de esta $X$ es $C_{x}$, que toma los valores siguientes:

\begin{tabular}{|l|l|l|l|l|l|l|l|l|}
\hline$X$ & 0,5 & 1 & 1,5 & 2 & 2,5 & 3 & 3,5 & 4 \\
\hline$C_{x}$ & 0,9863 & 0,98 & 0,93993 & 0,960 & 0,8663 & 0,82 & 0,7673 & 0,708 \\
\hline$X$ & 4,5 & 5 & 5,5 & 6 & 6,5 & 7 & 7,5 & 8 \\
\hline$C_{x}$ & 0,6423 & 0,57 & 0,4913 & 0,4060 & 0,31343 & 0,216 & 0,113 & 0 \\
\hline
\end{tabular}

Su representación gráfica aparece en la figura 2.

Fig. 2

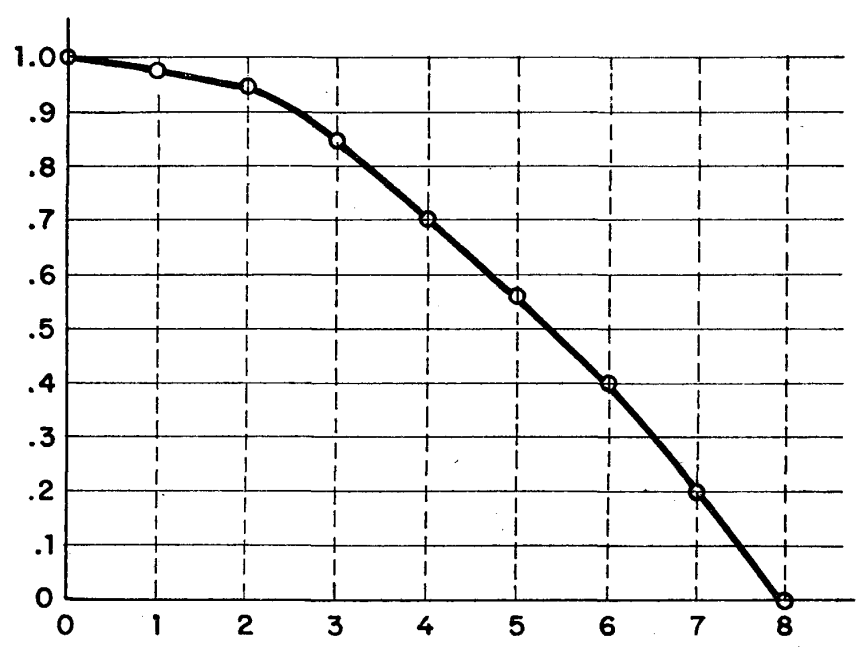

De la variación de $X$ con $V$ se puede obtener a su vez la variación de $C_{x}$ en función de $V$, para valores constantes de las resistencias características exigidas y obtenidas, y esta nueva función tiene la forma (fig. 3):

Fig. 3

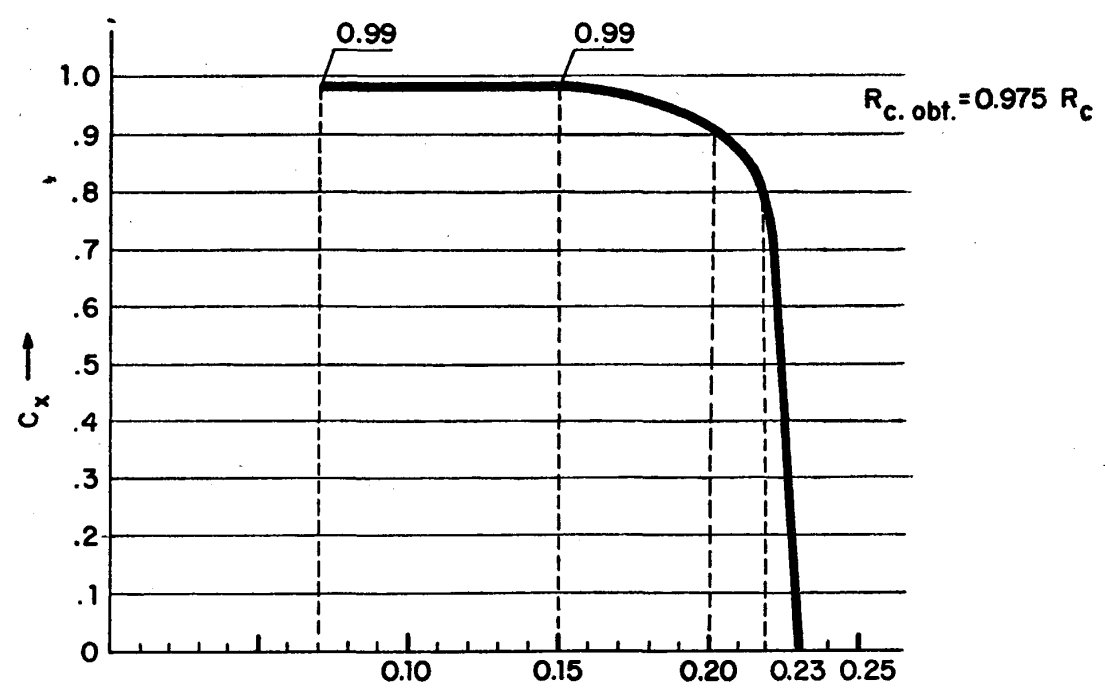


Por último, y en referencia al artículo 13, los valores de control que se indican son los necesarios para el caso en que $\alpha=1,64$; si tomamos $\alpha=1$ quedaría expresado de la siguiente forma:

1. Nunca se obtendrán más de TRES resistencias de control consecutivas de valor menor que la resistencia característica exigida.

2. La media de las resistencias de control de SEIS series consecutivas no será nunca menor que la resistencia característica exigida.

\section{Gomelusiomes}

Se ha presentado en proposición de Pliego de Condiciones para el suministro de hormigón preparado en centrales y suministrado en obra. Sería de gran interés que los técnicos en este campo expresaran sus opiniones para poder confeccionar sobre esta base $u$ otra más apropiada un Pliego de Condiciones en el que poder controlar un sector que tan gran auge está tomando de año en año.

\section{résumé̀ 0 summmanty}

\section{Proposition de cuhier des charges et de prix pour racquisition de béton fourni sur chamtier}

José M. López Saiz, ingénieur des Ponts et Chaussées

Cet article a pour but d'exposer un possible Cahier des Charges pour l'acquisition par contrat de béton prémélangé et de susciter, parmi les techniciens intéressés à ce sujet, une discussion de laquelle pourrait résulter l'élaboration d'un Cahier des charges appropriế aux exigences actuelles, étant donné que le nombre d'acquisitions par contrat de ce type de béton est très grand et, dans quelques villes,

La première partie de cet article porte simplement sur ledit Cahier des Charges. La deuxième partie fait un exposé des raisons qui ont amené à établir quelques-uns des articles, particulièrement ceux qui concernent les modalités de payement, ainsi qu'un commentaire sur le contrôle du béton en chantier, en désaccord avec les normes récemment publiées par le Ministère espagnol des Travaux Publics. On essaie a cet egard d envisager egalement un echange d'se dechnologie. D'ailleurs, Ministêre espagnol des Travaux Publics (1968).

\section{Proposal for specifications and purchosse pmice of concegte supplied on the site}

José M. López Saiz, civil engineer

This article seeks to establish a set of specifications for the purchase of premixed concrete, and also stimulate among those interested in this material a systematic discussion that may lead to the development of a specification suitable for current needs. The amount of ready mixed concrete that is being used is now very large, and in some regions it is the almost sole form of concrete used. In spite

The first part of the article merely cites the specification. In the second justifications are given for some of the headings of the specification, especially in regard to system of payment. Additionally, some comments are offered on the control of the conerete quality at the working site, which is in disagreement with the recently published regulation by the Ministry of Public Works. It is this branch of the techonology of concrete. Beyond this, it will be observed that the proposed specifications follow closely the current eInstructions of the Ministry of Public Works, 1968.

Vorschlag îber Vertrags-und Lieferbedingumgen fur dem aut dem Bauplatz geliefertem Beton

José M. López Saiz,

Diese Niederschrift hat den Zweck einen neuen Bedingungsbogen für den Einkauf von vorgemischtem Beton vorzuschlagen, in der Absicht, eine Diskussion unter den Fachleuten zu entfachen, welche zu einem aktuelleren Bedingungsbogenmodus führen könnte, um zeit-

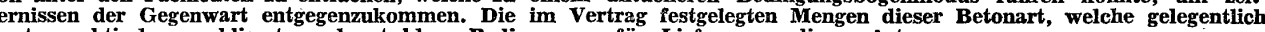
jede andere Betonsorte praktisch ausschliesst, verlangt klare Bedingungen für Lieferungen dieser Art.

Der erste Teil des Beitrages beschränkt sich auf die Darlegung des genannten Bedingungsbogens. In dem zweiten Teil wird die Formulierung einiger Klauseln -insbesondere bezüglich der Zahlungsbedingungen- klargestellt. Es wird auch die Aufsichtsfrage des Betons auf dem Bauplatz erötert und zwar in entgegengesetztem Sinne wie in der vor kurzen verabschiedeten Verfügung des M.O.P. (Ministerium für Offentliche Arbeiten) und in Anlahnung an die im Jahre 1968 verabschiedeten. Auch hier wird einen Meinungsaustausch der
Fachleute verfolgt. 